\title{
Rol patogénico del gen supresor de tumores PTEN en cáncer ovárico asociado a endometriosis
}

\author{
Adriana Castiblanco $\mathrm{G}^{1}$, Yumay Pires $\mathrm{N}^{1,2}$, \\ Ignacio Wistuba $0^{1,3}$, Erick Riquelme $S^{1}$, \\ Leonardo Andrade $\mathrm{M}^{1}$, Alejandro Corvalán $\mathrm{R}^{1}$. \\ Pathogenic role of PTEN tumor \\ suppressor gene in ovarian cancer \\ associated to endometriosis
}

Background: Endometrioid carcinoma and clear cell carcinoma of the ovary are associated to endometriosis. Somatic mutations of PTEN (10q23.3) are present in endometrial endometrioid carcinoma. Therefore, these mutations could be also present in ovarian tumors. Molecular studies show that solitary endometriotic cysts are monoclonal, have aneuploid DNA, have a loss of 9p,11q and 22q heterozygosity ( $\mathrm{LOH}$ ) and a higher cellular proliferation index of the epithelial component. Aim: To determine the cellular proliferation index using $\mathrm{Ki}$ 67, the immunohistochemical expression of PTEN and $\mathrm{LOH}$ in patients with ovarian endometriosis without atypia (EN), ovarian endometriosis with atypia (EA) and endometriosis with adjacent ovarian carcinoma (ET). Material and methods: Paraffin embedded samples of 37 endometrioid and clear cell carcinomas of the ovary (CC/CE), 15 solitary ovarian EN and 15 ovarian EA, were studied. Expression of Ki 67 and PTEN was measured by immunohistochemistry. LOH of 10q23.3 locus was measured by polymerase chain reaction. Results: Ki 67 was 5.5 and $2.3 \%$ in EA and EN, respectively ( $<<0.005$ ). There was a histological correlation between EA and a higher cellular proliferation index. PTEN was negative in 5 of $15 \mathrm{EN}, 9$ of $15 \mathrm{EA}$ and 30 of $37 \mathrm{CE} / \mathrm{CC}$. There was a correlation between LOH and loss of PTEN protein in EN, EA and ET (60\%). Conclusions: Negative expression on PTEN in EN; EA; ET and CE/CC is a manifestation of the inactivation of this gene. The mechanisms that cause this inactivation, must be elucidated (Rev Méd Chile 2006; 134: 271-8).

(Key words: Endometrial neoplasms; Endometriosis; Ovarian neoplasms; PTEN protein, human)

Recibido el 16 de diciembre, 2004. Aceptado el 25 de agosto, 2005.

Proyecto Becados PG09-02 Centro de Investigaciones Médicas, Escuela de Medicina, Pontificia Universidad Católica de Chile.

${ }^{1}$ Departamento de Anatomía Patológica, Escuela de Medicina, Pontificia Universidad Católica de Chile. ${ }^{2}$ Servicio de Anatomía Patológica, Clínica Alemana, Santiago, Chile ${ }^{3}$ Anderson Cancer Center, Houston, TX, USA.

Correspondencia a: Dra. Adriana Castiblanco. Departamento de Anatomía Patológica, Pontificia Universidad Católica de Chile. Lira 85, Santiago. Teléfono: 56(2) 3543628. Fax: 56(2)6395101.

E-mail: adrianitapat@starmedia.com 
$\mathrm{L}$ a endometriosis es la presencia de tejido endometrial fuera del útero. Los sitios más frecuentes de localización son ovarios, ligamentos uterinos, tabique recto-vaginal y peritoneo pélvico. La endometriosis es una enfermedad frecuente que afecta a $80 \%$ de las mujeres en edad reproductiva, con una mayor incidencia entre 35 y 44 años $^{1}$. Su prevalencia se estima en $10 \%$ a $15 \%$ con un riesgo de transformación maligna de $1,2 \%^{2,3}$. La asociación de endometriosis con carcinoma endometrioide o de células claras del ovario (CE/CC) se ha descrito en 15\% y 30\% de los casos, respectivamente ${ }^{4}$. En éstos la endometriosis puede estar adyacente al tumor, en el ovario contralateral o en otra parte. En 1925, Sampson describió por primera vez la asociación de endometriosis con carcinoma endometrioide del ovario, al observar en cortes histológicos focos de endometriosis adyacente al carcinoma ${ }^{1}$. En 1967, Scully describió esta misma asociación pero con carcinomas de células claras ${ }^{1}$. Sin embargo, en ambos casos, estos focos de endometriosis adyacentes al carcinoma presentaban características morfológicas diferentes a las de endometriosis sin tumor y fueron clasificadas como endometriosis atípica (EA) por dos grupos independientes $^{1,5}$. Recientes publicaciones ${ }^{7-11}$ han descrito alteraciones genéticas que apoyan el potencial preneoplásico de la endometriosis, como un mayor índice de proliferación celular en el componente epitelial independiente de la fase del ciclo del endometrio normal ${ }^{6}$, los análisis de clonalidad en quistes endometriósicos solitarios que demuestran monoclonalidad, el contenido de ADN aneuploide de las células epiteliales atípicas ${ }^{7-9}$ y la pérdida de heterocigocidad ( $\mathrm{LOH}$ ) de uno o más loci en los cromosomas 9p,11q y $22 q^{10}$. Debido a que estos eventos se han observado en focos de endometriosis adyacente a CE/CC, se considera a la endometriosis una lesión precursora de cáncer ovárico del tipo endometrioide y células claras ${ }^{1}$.

En el proceso de carcinogénesis ovárica están involucrados genes supresores de tumores (GST) y genes reparadores de ADN. Los GST codifican proteínas represoras de la proliferación celular no controlada y se inactivan por una combinación de eventos genéticos que comprometen ambas copias del gen, resultando en la ausencia de una proteína normal. Dichos eventos corresponden a mutación, deleción o metilación de ambos alelos del gen. Por otra parte, los genes reparadores de
ADN influyen en el proceso de carcinogénesis ovárica, porque su inactivación produce mutaciones de múltiples genes involucrados en el control de la proliferación celular, lo cual se manifiesta en el fenómeno de inestabilidad genética (IG) ${ }^{11}$.

Recientemente ha sido descubierto un GST, denominado PTEN (MMAC1/TEP1), localizado en el brazo largo del cromosoma 10q23.3 con función de fosfatasa, y que participa en la regulación del ciclo celular en la fase G1, adhesión celular y apoptosis $^{12}$. Utilizando métodos inmunohistoquímicos se ha logrado evaluar la pérdida de su expresión proteíca y es así como se han descrito mutaciones somáticas de PTEN en tumores de cerebro, mama, próstata, tiroides y, con mayor frecuencia, en carcinomas endometriales de tipo endometrioide ${ }^{21-23}$. En base a este último antecedente, se ha planteado la posibilidad de una vía común con tumores ováricos de tipo endometrioide, originados en focos de endometriosis y no del epitelio superficial del ovario ${ }^{13,14}$. En este escenario, en el presente trabajo evaluamos marcadores de proliferación celular e inactivación de PTEN en endometriosis sin atipia (EN), endometriosis atípica (EA) y endometriosis adyacente a tumor (ET) como eventos moleculares que sugieran una progresión de endometriosis a $\mathrm{CE} / \mathrm{CC}$ del ovario.

\section{MATERIALES Y MÉTODOS}

Casos clínicos. Se revisaron los informes de biopsias, del archivo del Departamento de Anatomía Patológica de la Pontificia Universidad Católica de Chile, en el período comprendido entre 1992 y 2002. Se incluyeron todos los tumores primarios del ovario con diagnóstico histológico de CE/CC del ovario. Se obtuvieron 37 tumores ováricos de pacientes que habían sido sometidas a ooforectomía por un tumor primario ovárico. Once casos tenían endometriosis adyacente a tumor (ET). Adicionalmente, se seleccionaron por características morfológicas 30 casos de endometriosis solitaria del ovario, 15 casos sin atipia (EN) y 15 casos de endometriosis atípica (EA). Todos los casos fueron revisados por un ginecopatólogo. Los criterios utilizados para definir endometriosis ovárica fueron la presencia de glándulas y estroma de tipo endometrial en la superficie del ovario o en la corteza ovárica, asociada a hemorragia, fibrosis e infiltrado inflamatorio de histiocitos con pigmento 
ceroide y hemosiderina con o sin lesión quística. Los criterios de inclusión de EA fueron aquellos casos de endometriosis solitaria con hipercromasia nuclear, aumento de la relación núcleo/citoplasma, estratificación, despenachamiento o aumento de la densidad glandular. La graduación histológica de los carcinomas endometrioides se consideró según la clasificación de la WHO (World Health Organization $)^{15}$ y para la graduación arquitectural se consideraron como G1 $(5 \%$ o menos patrón de crecimiento sólido), G2 (6-50\% patrón de crecimiento sólido) y G3 (>50\% patrón de crecimiento sólido) y se aumentó en un grado aquellos tumores G1 o G2 con acentuada atipia nuclear. Finalmente, en la evaluación de las áreas sólidas no se consideró focos con diferenciación escamosa o mórulas.

Inmunohistoquímica Ki 67 y PTEN. Se realizaron cortes histológicos de 6 micrones $(\mu \mathrm{m})$ de espesor de los tejidos fijados en formalina e incluidos en parafina de los casos seleccionados. Los cortes histológicos fueron sometidos a incubación con anticuerpo monoclonal anti Ki 67 (clon MIB 1; Dako), procesados según método S-ABC automatizado Biogenex i6000. Para la inmunohistoquímica de PTEN se usó el anticuerpo monoclonal anti PTEN (clon A2B1; Santa Cruz Biotechnology, Santa Cruz, CA), contra los aminoácidos 388-340 de la porción carboxi-terminal de PTEN. La especificidad de este anticuerpo ha sido documentada en otros estudios ${ }^{16}$. Los casos fueron examinados en forma ciega por dos observadores independientes. En cada caso se contaron los núcleos marcados en un total de 500 células usando aumento de 40X. El índice de proliferación celular fue medido por la evaluación del porcentaje de Ki 67 con tinción nuclear de las células de carcinoma y de endometriosis. La inmunotinción de PTEN se evaluó sin conocimiento de antecedentes clínicos ni patológicos, por visualización citoplasmática. Se consideró una tinción positiva cuando más de 10\% de las células estaban teñidas y la graduación de la intensidad fue: $0=$ ausente,$+=$ débil, $++=$ fuerte. Todos los casos con controles internos positivos y negativos.

Análisis de deleciones del locus 10q23.3 PTEN. El análisis de este locus se realizó mediante el estudio de pérdida de heterocigocidad (LOH) con técnica de amplificación por reacción de polimerasa en cadena (PCR) de ADN extraído de células obtenidas por microdisección de los casos de endometriosis y carcinomas, además de los controles de tejido normal (ovario y trompa de Fallopio). La microdisección se realizó en cortes de $6 \mu \mathrm{m}$ de tejidos fijados en formalina e incluidos en parafina y teñidos con hematoxilina y eosina (H-E) (Figura 1). La microdisección de los tejidos fue ejecutada bajo examen microscópico usando un micromanipulador hidráulico. La extracción de ADN se realizó agregándole al tejido microdisecado $100 \mu \mathrm{L}$ de solución de buffer con proteinasa $\mathrm{K}(300 \mathrm{mg} / \mathrm{mL})$ y se incubó a $55^{\circ} \mathrm{C}$ por $3 \mathrm{~h}$. La proteinasa $\mathrm{K}$ fue inactivada a $95^{\circ} \mathrm{C}$ por $10 \mathrm{~min}$ y las soluciones de ADN se almacenaron a $-20^{\circ} \mathrm{C}$ hasta su amplifica-

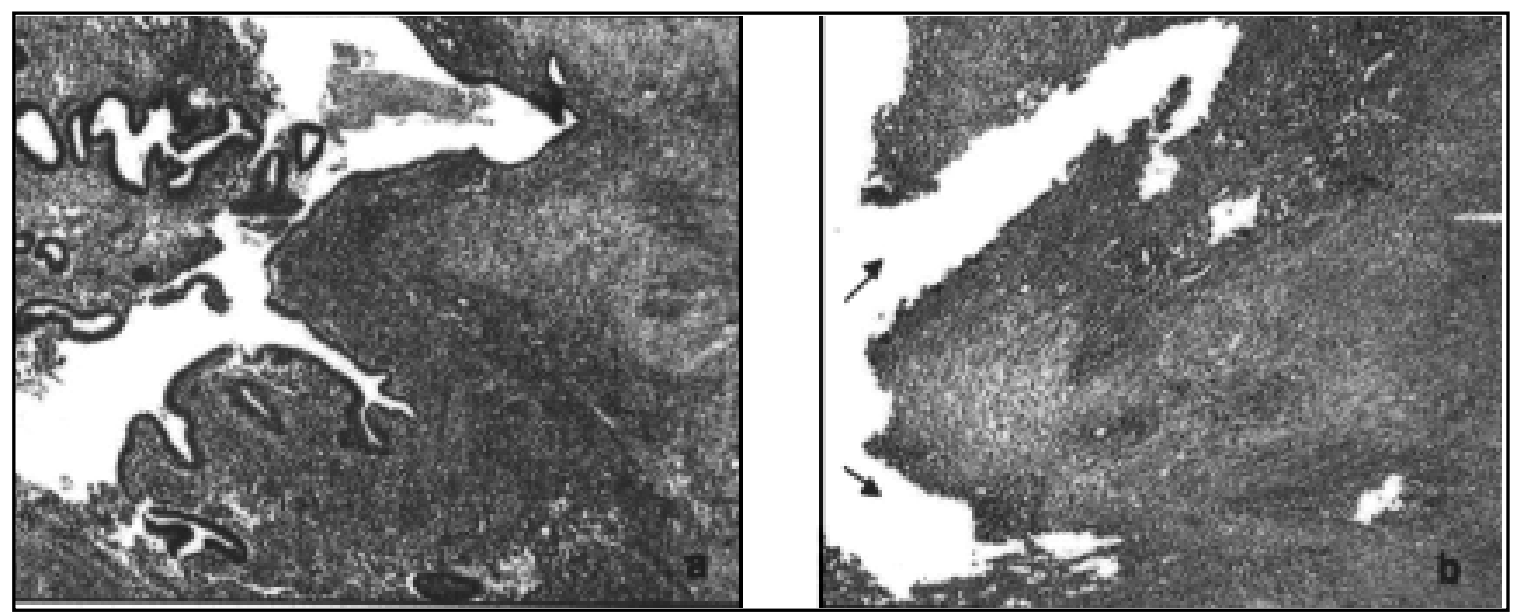

FIgura 1. Histología de una endometriosis sin atipia. En a) Endometriosis antes de microdisección (40X) y en b) Endometriosis después de microdisección (40X). 
ción. La amplificación por PCR se realizó en duplicado y con los controles respectivos. Las muestras fueron examinadas usando 5 marcadores de microsatélites del brazo cromosómico 10q (D10S215, AFM086wg9, D10S2491, D10S541, D10S185) utilizando $5 \mu \mathrm{L}$ de ADN extraído (concentración $10 \mathrm{ng}$ ) para cada marcador $\mathrm{P}^{32}$-marcado bajo condiciones estándar. El producto final del PCR fue separado en geles de poliacrilamida denaturantes al 6\% y luego visualizados a través de autorradiografía. La pérdida de heterogocidad (LOH), es decir la deleción de uno de los alelos paternos de un cromosoma se evaluó por detección visual de ausencia completa de un alelo en los casos informativos (heterocigotos).

Análisis de los datos. Los datos fueron analizados usando Visual Basic (Microsoft) específicamente diseñado para realizar diferentes cálculos en una hoja de Microsoft Excel, que incluían frecuencias de $\mathrm{LOH}$, índice FAL, frecuencias de corte, patrones de $\mathrm{LOH}$ por código de colores y análisis por grupos. Se realizó test exacto de Fisher y Wilcoxon rank para análisis estadístico. El valor de $\mathrm{p}<0,05$ fue considerado estadísticamente significativo.

\section{Resultados}

Se estudiaron 30 pacientes con endometriosis solitaria $(\mathrm{EN}=15$ y EA $=15), 37$ pacientes con CE/
CC $(\mathrm{CE}=29$ y $\mathrm{CC}=8), 11$ de las cuales tenían endometriosis adyacente a tumor (ET). El rango de edad de las pacientes con endometriosis solitaria (EN y EA) fue de 23 a 53 años (promedio de $39 \pm 7,7$ DS). El rango de edad de las pacientes con ET fue de 15 a 79 años (promedio de $52 \pm 5,8$ DS) y el rango de edad para las pacientes con CE/ CC fue de 15 a 79 años (promedio de $49 \pm 4,8$ DS). De los 29 CE, 21 ( $72 \%$ ) eran unilaterales y 20 (69\%) grado 3 de diferenciación histológica. Todos los CC fueron unilaterales. Los resultados del estudio inmunohistoquímico con Ki 67 se muestran en la Tabla 1 y Figura 2. El índice de proliferación celular en el epitelio de EA fue significativamente mayor que el observado en EN ( $\mathrm{p}<0,005$ ). Del mismo modo, se observaron

Tabla 1. Indice de proliferación celular (Ki 67) en endometriosis sin atipia, atípica y carcinoma endometrioide/ células claras

\begin{tabular}{|lccc|}
\hline & $\begin{array}{c}\mathrm{EN}^{\mathrm{a}} \\
(\mathrm{n}=15)\end{array}$ & $\begin{array}{c}\mathrm{EA}^{\mathrm{b}} \\
(\mathrm{n}=15)\end{array}$ & $\begin{array}{c}\mathrm{CE} / \mathrm{CC}^{\mathrm{c}} \\
(\mathrm{n}=37)\end{array}$ \\
\hline Epitelio glandular & 2,7 & 5,5 & 9,3 \\
Estroma & 2,4 & 2,7 & $\mathrm{~N} / \mathrm{E}$ \\
\hline
\end{tabular}

${ }^{\mathrm{a} E N}$ : Endometriosis sin atipia. ${ }^{\mathrm{b}} \mathrm{EA}$ : Endometriosis atípica. ${ }^{\mathrm{C}} \mathrm{CE} / \mathrm{CC}$ : carcinoma endometrioide/células claras. N/E no evaluada. ${ }^{a} \mathrm{~b} p<0,005$.

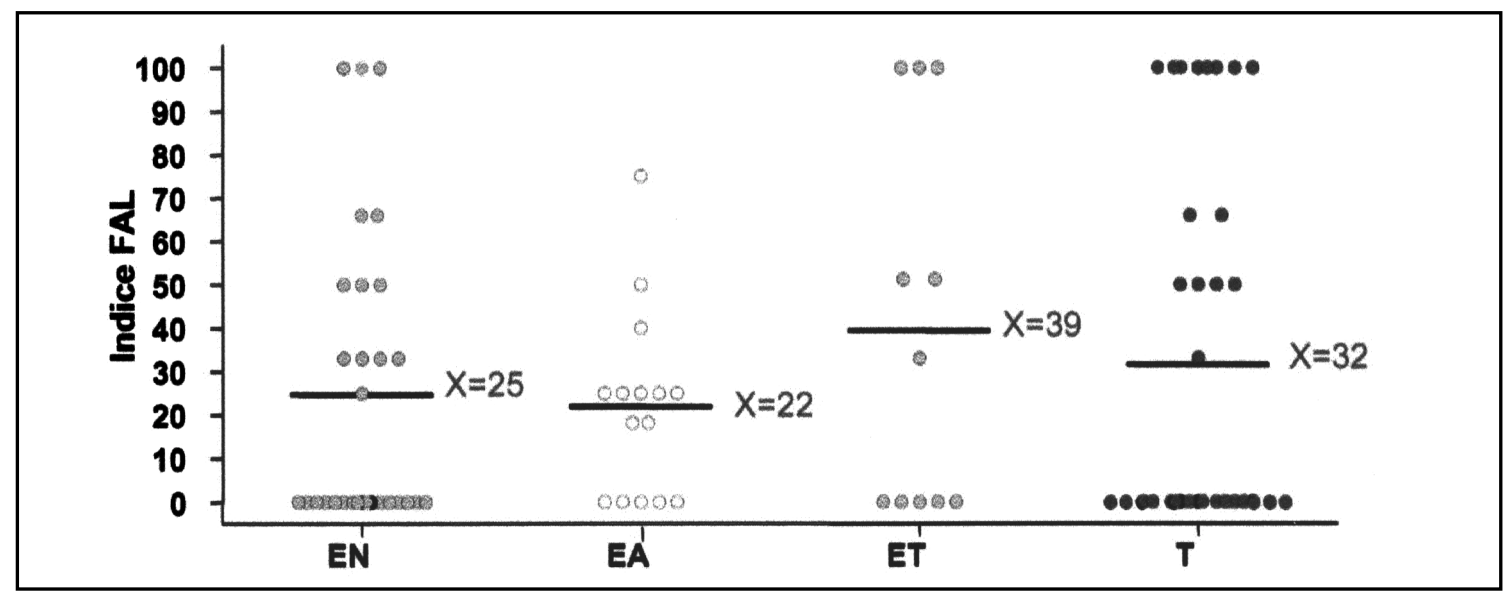

FiguRA 2. Indice de fracción de pérdida de alelos (FAL) en endometriosis y carcinoma endometrioide y células claras. $\mathrm{X}=$ Promedio del número de eventos de LOH en cada grupo. EN: endometriosis sin atipia; EA: endometriosis atípica; ET; endometriosis adyacente a tumor; CE/CC: carcinoma endometrioide/células claras. 
diferencias significativas entre el índice de proliferación de la EA y CE/CC ( $<<0,001)$. El índice de proliferación celular en el estroma no presentó diferencias significativas entre EN y EA. Los resultados del estudio inmunohistoquímico de PTEN se muestran en la Tabla 2 y Figura 2. De acuerdo a los criterios ya señalados, observamos una progresión de la negatividad de PTEN en los diferentes grupos de endometriosis y que es un evento relativamente frecuente en CE/CC. Entre los tumores con tinción positiva, $2(5 \%)$ casos fueron positivo débil $(+)$ y $5(14 \%)$ fueron positivo intenso $(++)$. No hubo correlación entre la tinción inmunohistoquímica de PTEN con el tipo histológico ni grado de diferenciación de los carcinomas endometrioides. Los resultados de los análisis de LOH se muestran en la Tabla 3. La pérdida de heterogocidad, con al menos un marcador de microsatélite se observó en 9/15 EN (60\%), 10/15 EA (66\%), 6/11 ET (55\%), y en CE/CC se observó con al menos un marcador de microsatélite en

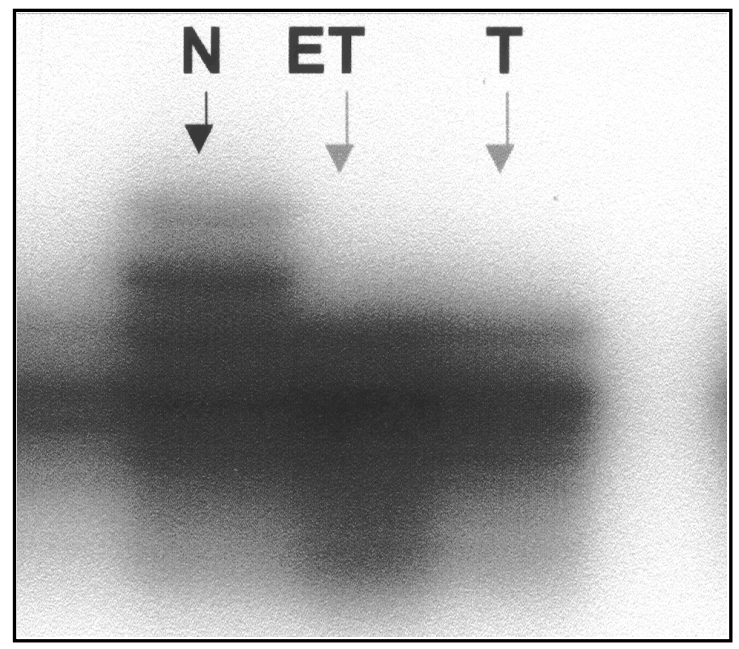

FIGURA 3. LOH en endometriosis adyacente a tumor y en carcinoma endometrioide. ET: endometriosis adyacente a tumor. $\mathrm{T}$ : carcinoma endometrioide. $\mathrm{N}$ : control.

Tabla 2. Expresión inmunohistoquímica de PT EN en endometriosis solitaria, adyacente a tumor y carcinoma endometrioide/ células claras

\begin{tabular}{|lcccc|}
\hline & \multicolumn{1}{c}{ EN $^{\mathrm{a}}$} & $\mathrm{EA}^{\mathrm{b}}$ & $\mathrm{ET}^{\mathrm{c}}$ & $\mathrm{CE} / \mathrm{CC}^{\mathrm{d}}$ \\
\hline PTEN - & $5(33 \%)$ & $9(60 \%)$ & $8(72 \%)$ & $30(81 \%)$ \\
PTEN + & $10(67 \%)$ & $6(40 \%)$ & $3(28 \%)$ & $7(19 \%)$ \\
Total & 15 & 15 & 11 & 37 \\
\hline
\end{tabular}

${ }^{a}$ EN: Endometriosis sin atipia. ${ }^{b}$ EA: Endometriosis atípica. ${ }^{\mathrm{c}} \mathrm{ET}$ : Endometriosis adyacente a tumor. ${ }^{\mathrm{d}} \mathrm{CE} / \mathrm{CC}$ : carcinoma endometrioide/células claras.

Tabla 3. Correlación entre la expresión inmunohistoquímica de PT EN y LOH 10q23.3 en endometriosis solitaria, adyacente a tumor y carcinoma endometrioide/ células claras

\begin{tabular}{|c|c|c|c|c|c|c|c|c|}
\hline \multirow[b]{2}{*}{ PTEN } & \multicolumn{2}{|c|}{$\mathrm{EN}^{\mathrm{a}}$} & \multicolumn{2}{|c|}{$\mathrm{EA}^{\mathrm{b}}$} & \multicolumn{2}{|c|}{$\mathrm{ET}^{\mathrm{C}}$} & \multicolumn{2}{|c|}{$\mathrm{CE} / \mathrm{CC}^{\mathrm{d}}$} \\
\hline & IHQ+ & IHQ- & $\mathrm{IHQ}^{+}$ & IHQ- & $\mathrm{IHQ}^{+}$ & IHQ- & $\mathrm{IHQ}^{+}$ & IHQ- \\
\hline $\mathrm{LOH}+$ & $5(50 \%)$ & $4(80 \%)$ & $6(100 \%)$ & $4(44 \%)$ & $1(33 \%)$ & $5(63 \%)$ & $2(29 \%)$ & $13(43 \%)$ \\
\hline LOH - & $5(50 \%)$ & $1(20 \%)$ & $0(0 \%)$ & $5(56 \%)$ & $2(67 \%)$ & $3(33 \%)$ & $5(71 \%)$ & $17(57 \%)$ \\
\hline Total & 10 & 5 & 6 & 9 & 3 & 8 & 7 & 30 \\
\hline
\end{tabular}

${ }^{a} \mathrm{EN}$ : Endometriosis sin atipia. ${ }^{\mathrm{b}} \mathrm{EA}$ : Endometriosis atípica. ${ }^{\mathrm{c}} \mathrm{ET}$ : Endometriosis adyacente a tumor. ${ }^{\mathrm{d}} \mathrm{CE} / \mathrm{CC}$ : carcinoma endometrioide/células claras. LOH: Pérdida de heterocigocidad. IHQ: Inmunohistoquímica. 
$30 \%(11 / 37)$ y con cualquiera de los 5 marcadores en 40\% (16/37). El promedio de LOH en EN y EA vs ET fue similar (63\% vs 55\%). El índice FAL definido como el número de eventos de LOH en una muestra dividido por el número total de marcadores informativos con ADN normal. El promedio de FAL en EN, EA y ET fue relativamente bajo $(0,28)$. Los resultados del índice FAL, se muestran en la Figura 3. El promedio de FAL no mostró diferencias significativas entre estos grupos ni correlación con las características histológicas de la endometriosis. La relación entre la pérdida de expresión inmunohistoquímica de PTEN y LOH de la región 10q23.3 se muestra en la Tabla 3. Se observó que en todos los grupos EN, EA, ET y CE/CC hay un número similar de casos con pérdida de expresión de PTEN y LOH, sin diferencias significativas entre ellos. Los resultados de LOH y PTEN en ET y CE/CC, por caso se muestran en la Tabla 4. Se observa una alta correlación en la pérdida de expresión de PTEN tanto en el tumor (CE/CC) como en la ET 63,6\% (7/11). Sólo 2 casos (casos 12 y 19) demostraron $\mathrm{LOH}$ tanto en el tumor como en la endometriosis

\section{Tabla 4. C orrelación entre la expresión inmunohistoquímica de PT EN y LOH 10q23.3 en 11 casos de endometriosis adyacente a tumor y carcinoma endometrioide/células claras}

\begin{tabular}{|lcccc|}
\hline & \multicolumn{2}{c}{ ETa } & \multicolumn{2}{c|}{ CE/CCb } \\
Caso & IHQ & LOH & IHQ & LOH \\
\hline 1 & - & - & - & - \\
12 & - & + & - & + \\
13 & - & - & - & - \\
14 & + & - & + & - \\
15 & + & + & + & - \\
17 & - & + & - & - \\
19 & - & + & + & + \\
21 & - & - & - & - \\
24 & - & + & - & - \\
25 & - & + & - & - \\
29 & + & - & - & - \\
\hline
\end{tabular}

${ }^{\mathrm{a} E T}$ : Endometriosis adyacente a tumor. ${ }^{\mathrm{b}} \mathrm{CE} / \mathrm{CC}$ : carcinoma endometrioide/células claras. IHQ: inmunohistoquímica PTEN. LOH: Pérdida de heterocigocidad. IHQ: Inmunohistoquímica. adyacente y 4 casos presentaron $\mathrm{LOH}$ sólo en la endometriosis adyacente al tumor (casos 15, 17, 24 y 25). Estos casos correspondían a focos de endometriosis que no estaban necesariamente en continuidad con el tumor y, por lo tanto, el tumor puede no haberse originado de ellos o el tumor haber sufrido una mutación independiente del gen PTEN, por lo que no se observa LOH.

\section{DisCUSIÓN}

La endometriosis como lesión precursora de carcinoma endometrioide y de células claras del ovario ha sido ampliamente estudiado. Sin embargo, en mujeres chilenas no se conocen datos reportados hasta el momento. Nuestros resultados muestran una diferencia de 10 años de edad entre las pacientes con endometriosis solitaria (EN y EA) y aquellas con CE/ CC, similar a lo descrito en un estudio previo con seguimiento de 11,4 años a mujeres con historia de endometriosis ${ }^{6}$. Otros estudios han demostrado que el riesgo de desarrollar cáncer ovárico, comparado con la población normal, es de 1,2\% a 4,2\% después de 10 años de historia de endometriosis ${ }^{3}$. El hallazgo de un mayor índice de proliferación celular en el epitelio de la EA está en relación con las características morfológicas descritas por LaGrenade et al $^{5}$ y permitirían considerar a la EA como un grupo diferente de la EN con características propias y comportamiento diferente. La pérdida de la expresión inmunohistoquímica de PTEN en los diferentes grupos de endometriosis muestra diferencias significativas entre EN y CE/CC (p 0,002) y entre todos los grupos de endometriosis y CE/CC (p 0,016), lo cual sugiere una participación de este gen en la transformación maligna de la endometriosis. La frecuencia de $\mathrm{LOH}$ en 10q23.3 en la endometriosis solitaria (EN y EA) es mayor que la observada en 9p, 11q y 22q, según lo reportado en la literatura ${ }^{14-17}$. La observación de LOH en la EN indica que esta lesión con características histológicas benignas ya tiene alteraciones moleculares similares a las de EA y ET y probablemente contribuya a la selección clonal en la progresión neoplásica. En nuestra serie no encontramos diferencias significativas de LOH 10q23.3 en endometriosis solitaria (EN y EA) y CE/CC. La relación entre LOH y PTEN negativa no mostró diferencias entre EN, EA y ET. LOH es un evento que se observa en todos los grupos pero que no 
aumenta con la pérdida de expresión de proteína de PTEN. Estos hallazgos sugieren que otros mecanismos adicionales deben participar en la inactivación de PTEN. En este sentido es posible que mecanismos como la hipermetilación aberrante de la región promotora de PTEN sea uno de estos potenciales mecanismos adicionales. La hipermetilación aberrante ha sido descrita en carcinomas endometriales y ováricos asociados a inestabilidad de microsatélites y enfermedad metastásica ${ }^{17,18}$. Adicionalmente, se han demostrado alteraciones en la expresión inmunohistoquímica de bcl-2 y p53 en endometriosis solitaria, ET y CE/CC, sugiriendo que estos genes estarían involucrados en la transformación maligna de la endometriosis $^{19}$. También se han descrito diferencias

\section{REFERENCIAS}

1. Kurman RE. Blaustein's Pathology of the Female Genitale Tract. Fourth Edition ed.

2. Fukunaga M, Nomura K, Ishikawa E, Ushigome $\mathrm{S}$. Ovarian atypical endometriosis: its close association with malignant epithelial tumours. Histopathology 1997; 30: 249-55.

3. Brinton La, Gridiey G, Persson I, Baron J, Bergevist A. Cancer risk after a hospital discharge diagnosis of endometriosis. Am J Obstet Gynecol 1997; 176: 572-9.

4. Fetiey KM, Welus M. Precursor lesions of ovarian epithelial malignancy. Histopathology 2001; 38: 87-95.

5. LaGrenade A, Silverberg SG. Ovarian tumors associated with atypical endometriosis. Hum Pathol 1988; 19: 1080-4.

6. Nisolle M, Casanas-Roux F, Donnez J. Immunohistochemical analysis of proliferative activity and steroid receptor expression in peritoneal and ovarian endometriosis. Fertil Steril 1997; 68: 9129.

7. Nilbert M, Pejovic T, Mandahl N, Iosif S, Willen H, Mitelman F. Monoclonal origin of endometriotic cysts. Int J Gynecol Cancer 1995; 5: 61-3.

8. Thomas EJ, Campbell IG. Molecular genetic defects in endometriosis. Gynecol Obstet Invest 2000; 50 Suppl 1: 44-50.

9. Yano T, Jimbo H, Yoshikaina H, Tsutsumi O, Taketani Y. Molecular analysis of clonality in ovarian endometrial cysts. Gynecol Obstet Invest 1999; 47 Suppl 1: 41-45; discussion 46. entre endometriosis quística y no quística, observándose una sobreexpresión de colágeno IV y una baja expresión de bcl-2 y metaloproteinasa IX en endometriosis quística ${ }^{20}$. Tomados en conjunto, nuestros datos muestran que la EA presenta características morfológicas que se relacionan con un mayor índice de proliferación celular. Nuestros resultados confirman la alta frecuencia de $\mathrm{LOH}$ (10q23.3) y de pérdida de expresión de PTEN en EN, EA y ET. Sin embargo, el número de eventos de LOH no mostró diferencias significativas. Por lo tanto, se hace necesario el estudio de otros mecanismos de inactivación como metilación y mutación que permitan sustentar un modelo secuencial de progresión de endometriosis a carcinoma.

10. Jiang X, Morland SJ, Hitchcock A, Thomas EJ, Campbell IG. Allelotyping of endometriosis with adjacent ovarian carcinoma reveals evidence of a common lineage. Cancer Res 1998; 58: 1707-12.

11. Vogelstein B, Kinzler K. The Genetic Basis of Human Cancer. New York: McGraw-Hill, 2001.

12. Risinger Ji, Hayes AK, Berchuck A, Barrett JC. PTEN/MMAC1 mutations in endometrial cancers. Cancer Res 1997; 57: 4736-8.

13. Sato N, Tsunoda H, Nishida M, Morishita $\mathrm{Y}$, TAкimoto Y, Kubo T et Al. Loss of heterozygosity on 10q23.3 and mutation of the tumor suppressor gene PTEN in benign endometrial cyst of the ovary: possible sequence progression from benign endometrial cyst to endometrioid carcinoma and clear cell carcinoma of the ovary. Cancer Res 2000; 60: 7052-6.

14. Obata K, Hoshiai H. Common genetic changes between endometriosis and ovarian cancer. Gynecol Obstet Invest 2000; 50 Suppl 1: 39-43.

15. Jaffe ES, Harris NL, Stein H, Vardiman JW. Pathology and Genetics of Tumours of the Breast and Female Genital Organs. Lyon: IARC Press, 2001.

16. Kanamori Y, Kigawa J, Itamochi H, Shimada M, Takahashi M, Kamazawa S et al. Correlation between loss of PTEN expression and Akt phosphorylation in endometrial carcinoma. Clin Cancer Res 2001; 7: 892-5.

17. Salvesen HB, MacDonald N, Ryan A, Jacobs IJ, Lynch ED, Akslen LA et al. PTEN methylation is associated with advanced stage and microsatellite 
instability in endometrial carcinoma. Int J Cancer 2001; 91: 22-6.

18. Fu Y, Campbell EJ, Shepherd TG, Nachtigal MW. Epigenetic regulation of proprotein convertase PACE4 gene expression in human ovarian cancer cells. Mol Cancer Res 2003; 1: 569-76.

19. Nezhat F, Cohen C, Rahaman J, Gretz H, Cole P, KaliR T. Comparative immunohistochemical studies of bcl-2 and p53 proteins in benign and malignant ovarian endometriotic cysts. Cancer 2002; 94: 2935-40.

20. Nezhat FR, Kalir T. Comparative immunohistochemical studies of endometriosis lesions and endometriotic cysts. Fertil Steril 2002; 78: 820-4.

21. Liu WG, Jame CD, Frederik L, Alderete BE, Jenkins RB. PTEN/MMAC1 mutations and EGFR amplification in glioblastomas. Cancer Res 1997; 57: 5254-7.
22. Cairs P, Okami K, Halachmi N, Esteller M, Herman JC, IsAacs WB ET AL. Frequent inactivation of PTEN/MMAC1 in primary prostate cancer. Cancer Res 1997; 57: 4997-5000.

23. Liaw D, Marsh DJ, Li J, Dahia PLM, Wang SI, Zheng ZM ET AL. Germline mutations of the PTEN gene in Cowden disease, and inherited breast and thyroid cancer syndrome. Nat Genet 1997; 16: 647.

24. Orbo A, Nilsen M, Arnes M, Pettersen I, Learsen K. Loss of expresión of MLH1-MSH2, MSH6 and PTEN Related to Endometrial Cancer in $68 \mathrm{~Pa}$ tients with Endometrial Hyperplasia. Int J Gynecol Pathol 2003; 22: 141-8.

25. Salvesen HB, Stefansson I, Kalvenes MB, Das S, AKslen LA. Loss of PTEN expression is associated with metastasic disease in patients with endometrial carcinoma. Cancer 2002; 94: 2185-91. 